\title{
Development and Implementation of Graphical User Interface for Image Preprocessing using Matlab
}

\author{
Shabari Shedthi B. \\ Department of CSE \\ NMAMIT, Nitte \\ Karnataka, India
}

\author{
Surendra Shetty, PhD \\ Department of MCA \\ NMAMIT, Nitte \\ Karnataka, India
}

\author{
M. Siddappa, PhD \\ Department of CSE \\ SSIT, Tumkur \\ Karnataka, India
}

\begin{abstract}
Image pre-processing is a basic step for any image based applications. It is a technique to enhance quality of the images captured from cameras or sensors, aircrafts and space probes or pictures taken for various applications in normal day-to-day life. The accuracy of this technique must be significantly high in order to ensure the success of the subsequent steps. This intended software has the intention to make image preprocessing more efficient and interactive so that the user has clear idea about the outcome of each manipulation. In the intended application, one can manipulate the corrupted image to get the meaningful, desired output by applying various techniques provided in the software. The outcome of the intended application is obtained immediately and reduces the queue time to perform manipulations for the experienced user. If the user is a beginner, then he can go for trial session to try all the techniques and understand their outcome.
\end{abstract}

\section{Keywords}

Image preprocessing; GUI (Graphical User Interface); Edge Detection; User Friendly Interface; Filters; Segmentation; Morphological Processing.

\section{INTRODUCTION}

Image is composed of finite number of picture elements, called as pixel. The naked eye cannot understand the pixel information. Images can be damaged, corrupted, tampered due to many reasons. It may be due to inadvertent movement of the camera while capturing the picture, camera equipment problem, and/or noise added during transmission of the image etc. In many of the cases it is required that the original image be without any noise so that the desired information is unadulterated. In the intended interactive software, user can examine the image, try manipulations and the respective result will be immediately shown to the user so that he can decide on what has to done be to the image, to extract the intended data out of it. In this application, image is browsed from already captured images which need to be processed. The captured image would be processed using many algorithms to get meaningful information out of it.

\section{LITERATURE SURVEY}

The different perceptions regarding image preprocessing techniques by various authors along with the related work is discussed in this section.

Singh et.al [1] explained that aim of the image processing is the better recognition of interested object. Find the suitable features which are used for distinguish the objects from its background. There are different preprocessing techniques like edge detection, filtration, segmentation, morphological operation etc.
Histogram of an image is useful to analyze appearance of the image through gray levels. Histogram equalization helps to enhance the image [2]. Image enhancement is useful for reduce the noise from image, enhancement the quality of the dark image and to highlight the edges [3]. The aim of histogram equalization is to evenly spread out the contrast to throughout the entire available dynamic range from 0 to 1 . Adhikari et al [4] presented a technique to differentiate the interested region from the background image.

Edge detection is a technique to identify the edges in an image. In image edges defines the boundaries between the regions which helps for image segmentation for highlighting the interested region and object recognition. According to the Nadernejad et al [5], Canny's method is preferred because it produces single pixel thick, continuous edges. Maini[6] discussed the different edge detection techniques which are most commonly used in Laplacian based and Gradient-based edge Detection under noisy conditions. It is necessary to get rid of noise before succeeding image process tasks in a contaminated image. The occurrence of noise will spoil the knowledge or information contained within the original images. Median filter is used for removing or reducing the error. Fuzzy rule based Median filter gives better solution [7]. Spatial domain filters have been employed very effectively in removing different types of noise, in many image processing applications [8]. Alshamarti et al [9] proved that median filter with threshold technique is better noise removal than the only mean filter in many cases. Al-amri et al [10] proposed different blurring techniques to restore the image features and remove blurriness in the image.

Nida et.al [11] surveyed about different segmentation techniques. In digital image processing image segmentation has a very important role. The main goal of image segmentation is to clearly differentiate the object and the background in an image. Segmentation means to partition or highlight an image based on drastic changes in intensity value, over viewed about of different segmentation techniques like clustering, Histogram etc. and also listed different applications of image segmentation. Nikitha et.al [14] discussed about various color images and how to evaluate the performance also mentioned in paper. Saini and Arora [15] explained in detail about edge based segmentation and region based segmentation. Raid et al [16] explained and implemented different morphological operations like erosion, dilation, opening, closing etc.

\section{GRAPHICAL USER INTERFACE}

Image quality can be identified by visual inspection but this process is inefficient and time consuming. Therefore there is a need to preprocess the image before evaluation of the image. Preprocessing will increase the quality of the image. 
Graphical User Interface tool makes the image preprocessing job easier and faster.

The image preprocessing is the combination of many algorithms or methods to obtain desired output. Preprocessing is a method of transforming a source image to new image. This preprocessed image is almost similar to the source image, but differs in certain quality aspects like improved contrast, reduced noise, deblur etc. Image processing through GUI makes the things simple and faster rather than entering commands. Preprocessed image gives better accuracy in pattern recognition and in classification.

Some of the important processing techniques which are necessary for preprocessing and to reduce noise if produced during acquirement or transmission used in this intended software are -

\section{Edge detection \\ 2. Filtration \\ 3. Segmentation \\ 4. Morphological operation \\ 5. Image formats}

It also includes some of the basic editing tool such as cropping, rotation, histogram modification and boundary extraction.

\subsection{Edge detection}

Edge detection is an image processing technique which helps to characterize the boundaries of objects within the image. Edge detection is part of image segmentation. It helps to remove the unnecessary information by preserving the important properties in an image. Since edge detection is the important steps of image processing, it helps to object detection. But it is difficult to have a good understanding of edge detection algorithms.

This proposed work provides the option to check various edge detection operations. Methods used in this are Sobel, Canny, Robert, prewitt, Zero cross and LoG (Laplacian of Gaussian).

\subsection{Filtrering}

Filtering is a technique used to remove the undesirable features or characteristics from the image. In image processing filtering is a pre-processing technique which includes smoothing, sharpening, and edge enhancement. Filtering is a neighbourhood operation, in which the value of the output image is determined by the neighbourhood values. This is obtained by applying some algorithm or transformation function to the values of the pixels in the neighbourhood of the corresponding input pixel. Here 15 distinct types of filters are implemented so that user can directly get the filtered image by just pressing one button.

\subsection{Segmentation}

The most important part of image pre-processing is segmentation. Segmentation is used to find out certain object in the image by subdividing the image into its constituent regions or objects. It divides the image into meaningful structures and reduces the information so that given image is easy to analyse. Obtained compact representation of image will provide useful information about the surface scene.

Segmentation technique is widely used for compression of images, editing, estimating the boundary from the image and recognition of objects. Segmentation can be classified based on type of algorithms used, that is Region based segmentation, Edge based segmentation, threshold, Feature based clustering, and Model based segmentation, whereas this work introduces four popular methods of segmentation, they are Watershed, Otsu, K-means clustering, and texture segmentation etc. It has a large scope in object or pattern recognition.

\subsection{Morphological operation}

Morphological operation is helps to extract the image component which is useful for image representation and description of boundaries. Mathematical morphological tool aims of removing the imperfections in structure of the image. Morphological operations used are Erosion, Dilation, Opening, Closing and filling holes. Through this minute unnecessary items can be removed or gap can be filled.

\subsection{Image format}

Given image can be expressed in many formats based on requirements. Normally image will be in RGB format. There are many formats which changes the visual appearance of images so that objects which are not identified using naked eyes can be categorized in different color format or colour spaces. Different colour formats used are gray scale, HSV, HSI and binary.

\section{RESULT AND DISCUSSIONS}

It is observed that image preprocessing is the foundation for any further manipulation and it is very important step in classification. Before pattern identification each image has undergo different preprocessing steps for better recognition. Selection of suitable preprocessing technique is one of the challenging tasks in pattern recognition.

Current work includes many preprocessing techniques and is implemented using GUI tool of matlab, which provides effective user interface. In the intended application, different techniques of image preprocessing is put together for effective user interface. The main screen provides a button to browse an image. Browsed image will display on left top of the screen and preprocessed image will be displayed on below the input image. Different options for preprocessing techniques we can choose from right side of the screen. Processed can be saved through save as button for further processing.

Histogram is a graph, it tells about occurrence of the grey level in the image. The appearance of an image can be analyzed from its histogram. In a dark image, the grey levels would be towards at the lower end. In bright image, the grey levels are moved to upper end. In a well contrasted image, the grey levels are spread out over the entire range. Histogram explains about nature of the image. Histogram of the chosen image is shown in Fig.1. 


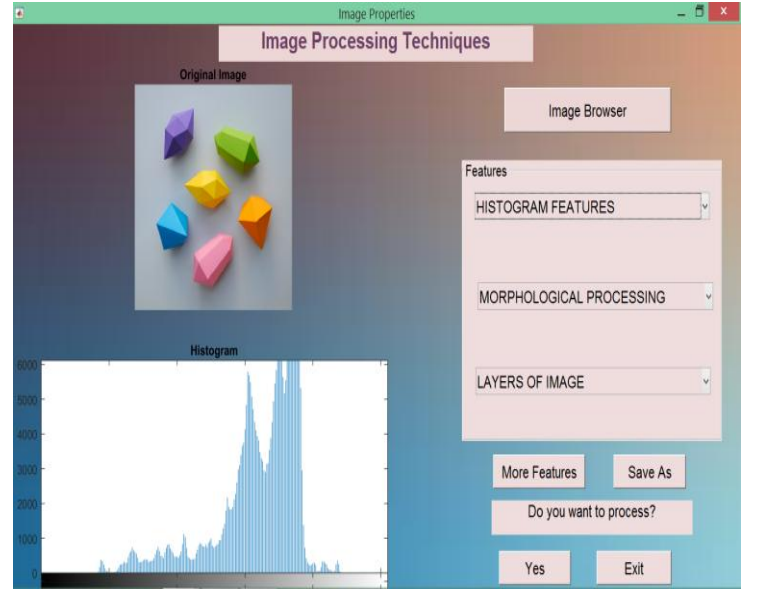

Fig.1 GUI page which helps to understand different image features. Histogram of selected image is shown

Edge detection is important step in object recognition. Edge has large variant of pixels compared to its other area. Different edge detection techniques behave differently in various scenarios. Prewitt filter very sensitive to noise. This is the major drawback of it. The performance of the canny algorithm depends mainly on adjustable parameters like standard deviation and threshold values. This algorithm is computationally more expensive compared to sobel, prewitt and robots algorithm.

After comparing various types of edge detection algorithms, it is found that canny technique is better than other edge detector for selected image. That is shown in figure 2 .

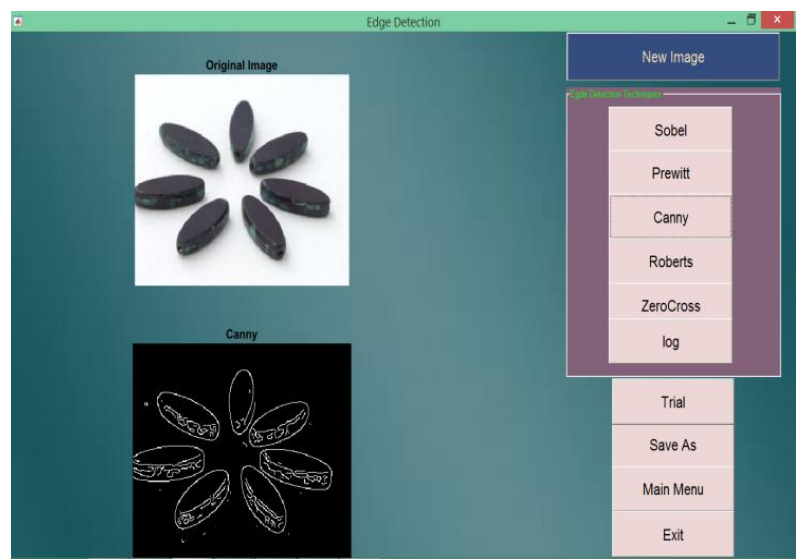

Fig.2 GUI showing Edge Detection using Canny algorithm

Filters provide clarity to visual interpretation of images. This filtered image is used as a precursor to further digital processing. Implementation of several different filtration techniques like mean, median, wiener etc are put together so that user can select which technique suits for the browsed image. Median filter preserves edges in an image while reducing noise shown in fig.3. Different filters are tested for a given contaminated images to reduce the noise.

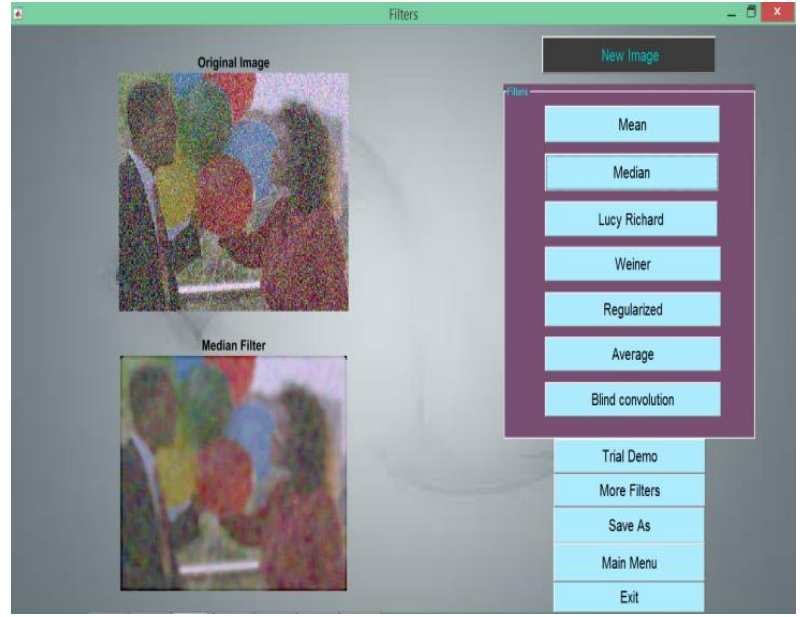

Fig.3 GUI for filters and Median Filter applied on chosen image

Segmentation is the most important part in image processing. It divides entire image into several parts based on intensity. This is very useful to highlight the required regions, which is meaningful and easier for further analysis. The main motto of segmentation is to reduce the information for easy analysis. Segmentation is used in image either to distinguish objects or to partition an image onto the related regions. It responds more quickly to human eye and produces result accurately so as the related scene is concerned. Clustering algorithm involved in intended application is advantageous because the classification is simple and easy to implementation for clusters.

A cluster is a collection of related data objects that are similar to one another within the same cluster and dissimilar to the object in other cluster. Texture segmentation analysis place an important role in many tasks such as scene classification, shape determination etc. Texture segmentation helps to identify the same texture features, so that further analysis can be performed on the respective regions alone. Watershed algorithm gives complete division of the image. It has low computational complexity and it suffers from over segmentation.

It was observed that using segmentation technique the object and the background are clearly differentiated (Fig.4).

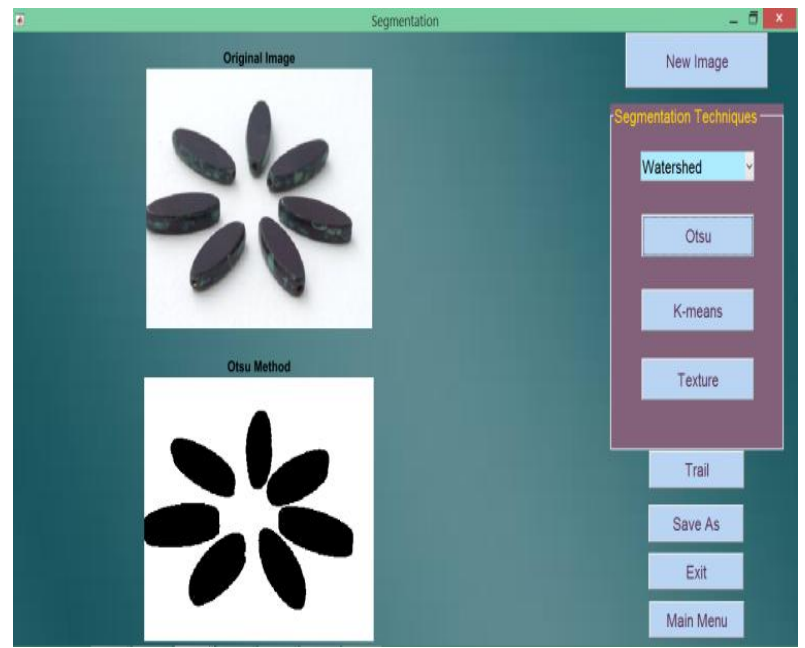

Fig.4 GUI for Segmentation and Otsu algorithm is applied on the chosen image 
A morphological operation helps to identify element fits within the neighborhood. Erosion process will allow thicker lines to get skinny. Dilation is the opposite operation of the erosion. Figures that are very lightly drawn object get thick when dilated.

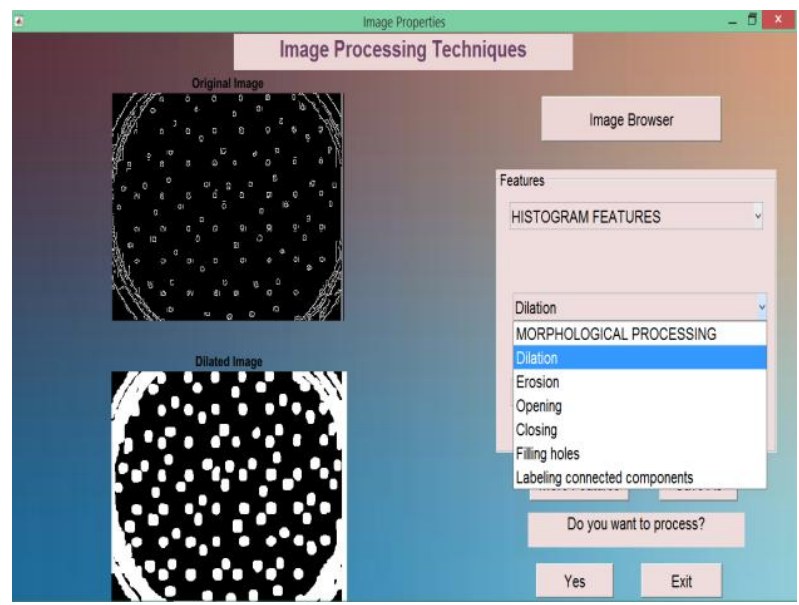

Fig.5 GUI for Morphological processing and Dilation algorithm applied on chosen image.

Erosion shrinks an image and Dilation expands it. Opening operation removes the outer tiny hairline leaks. The side effect is that it rounds off things so sharp edges start to disappear. When the morphological operations were added to the binary image, the imperfections were removed (Fig.5).

An RGB image sometimes is also called as true color image. It is stored as an $m$-by- $n$-by- 3 data array. It defines red, green, and blue color components for each individual pixel.

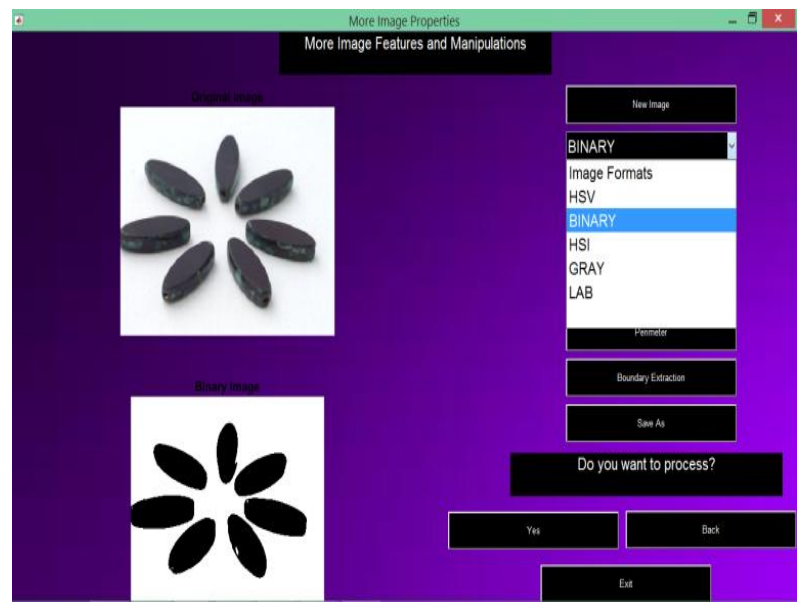

Fig.6 GUI for Image formats and shows binary image of chosen image

An intensity image is a data matrix, whose values represent intensities within some range. An intensity image is represented as a single matrix, with each element of the matrix corresponding to one image pixel. In essence, intensity images are treated as indexed images. According to the requirement, format of the image can be changed (Fig.6).

\section{CONCLUSIONS}

Intended application is built to test different image preprocessing techniques on the chosen image. Different properties of the selected image can be studied and analyzed visually. Selecting the best image preprocessing technique is very challenging task because it depends on type of image.
Through this GUI different image processing techniques can apply for one image and can compare which technique is most suitable for that image.

Implementation of image preprocessing through this software avoids manual work of entering the commands each and every time to apply one algorithm after another on the chosen image. Also this interface gives more interactive options to experience different techniques. This software has integrated five techniques i.e Filtration, Edge detection, segmentation, Image formats and Morphological processing in one platform. This helps the beginner to experience new image preprocessing techniques and analyze the output image. Through this GUI user can select most suitable technique for their data before taking into further processing so they can achieve high accuracy.

In future we can extend the scope of this work to fix the needy size of image for further processing and further can apply standard machine learning techniques to design a decision making modules.

\section{REFERENCES}

[1] Krishna Kant Singh, Akansha Singh, "A Study of Image Segmentation Algorithms for Different Types of Images”, IJCSI, vol. 7, Issue 5, Sep 2010.

[2] R. Krutsch, \& D. Tenorio, "Histogram Equalization", Freescale Semiconductor, Document Number AN4318, Application Note, Jun 2011.

[3] K. K Lavania, Shivali, R. Kumar, "Image Enhancement Using Filtering Techniques" International Journal on Computer Science and Engineering (IJCSE), vol. 4, No. 01, Jan 2012.

[4] Subhajit Adhikari, Joydeep Kar, Jayati Ghosh Dastidar," An automatic and efficient foreground object extraction scheme", International Journal of Science and Advanced Information Technology, Apr 2014.

[5] Ehsan Nadernejad, Sara Sharifzadeh, Hamid Hassanpour," Edge Detection Techniques: Evaluations and Comparisons, Applied Mathematical Sciences", vol. 2, no. 31, 1507 - 1520, Dec 2007

[6] Raman Maini and Himanshu Aggarwal," Study and Comparison of Various Image Edge Detection Techniques, IJIP, vol. (3), Issue (1), Feb 2009.

[7] Kh. Manglem Singh, "Fuzzy Rule based Median Filter for Gray-scale Images", Journal of Information Hiding and Multimedia Signal Processing, vol. 2, Number 2, Apr 2011.

[8] R. Oten and R. de Figueiredo," Adaptive AlphaTrimmed Mean Filters under Deviations from Assumed Noise Model", IEEE Trans. Image Processing, vol. 13, No. 5, pp. 627-639,Apr 2004.

[9] H.A. Alshamarti, Ali K. Hussein, B.A. Almayahi, "Application of median filter with the threshold technique to reduce and remove gaussian noise on the image edges produced by sobel operator", International Journal Of Computer Engineering and Technology(IJET), vol. 4, Issue 6, Dec 2013.

[10] Mr. Salem Saleh Al-amri, Dr. N.V. Kalyankar and Dr. Khamitkar S.D, "Deblured Gaussian Blurred Images", Journal of computing, vol. 2, Issue 4, Apr 2010. 
[11] Nida M.Zaitoun, musbah J. Aqel "survey on Image Segmentation Techniques", International Conference on Communication, Management and Information Technology (ICCMIT), Elsevier, 2015

[12] Rafael C. Gonzalez, Richard E. Woods, "Digital Image Processing", 2nd Ed. Beijing: Publishing House of Electronics Industry, 2007.

[13] Satish Kumar, Raghavendra Srinivas, "A Study on Image Segmentation and its Mae4dethods", International Journal of Advanced Research in Computer Science and Software Engineering, vol. 3, Issue 9, Sep 2013.

[14] Nikita Sharma, Mahendra Mishra, Manish Shrivastava, "Colour Image Segmentation techniques and issues: an approach”, International Journal of Scientific \& Technology Research vol. 1, Issue 4, May 2012.

[15] Sujata Saini and Komal Arora,"A Study Analysis on the Different Image Segmentation Techniques", International Journal of Information \& Computation Technology. ISSN 0974-2239 vol 4, Dec 2013.

[16] A.M Raid, W.M.Khedr, M.A.El-dosuky ,Mona Aoud, "Image Restoration Based on Morphological Operations", International Journal of Computer Science, Engineering and Information Technology (IJCSEIT), Vol. 4, No.3, June 2014. 